Mexico. Microb Drug Resist 2003;9:33-38.

10. Bobadilla-del-Valle M, Ponce-de-Leon A, Arenas-Huertero C, Vargas-Alarcon G, KatoMaeda M, Small PM, et al. rpoB Gene mutations in rifampin-resistant Mycobacterium tuberculosis identified by polymerase chain reaction singlestranded conformational polymorphism. Emerg Infect Dis 2001;7:1010-1013.

II. Molina-Torres CA, Moreno-Torres E, Ocampo-Candiani J, Rendon A, Blackwood K, Kremer K, et al. Mycobacterium tuberculosis spoligotypes in Monterrey, Mexico. J Clin Microbiol 2010;48:448-455.

12. Garza-Gonzalez E, Gonzalez GM, Renteria A, Cruz-Pulido W, Rivera G, Bocanegra-Garcia V.

A pyrosequencing method for molecular monitoring of regions in the inhA, ahpC and rpoB genes of Mycobacterium tuberculosis. Clin Microbiol Infect 2010;16:607-612.

13. Zenteno-Cuevas R, Zenteno JC, Cuellar A, Cuevas B, Sampieri CL, Riviera JE, et al. Mutations in $r p o B$ and katG genes in Mycobacterium isolates from the Southeast of Mexico. Mem Inst Oswaldo Cruz 2009; 104:468-472.

14. Ramaswamy SV, Dou SJ, Rendon A, Yang Z, Cave MD, Graviss EA. Genotypic analysis of multidrug-resistant Mycobacterium tuberculosis isolates from Monterrey, Mexico. J Med Microbiol 2004;53:107-I I3.

15. Jin DJ, Gross CA. Mapping and sequencing of mutations in the Escherichia coli rpoB gene that lead to rifampicin resistance. J Mol Biol 1988;202:45-58.

16. Telenti A, Imboden P, Marchesi F, Lowrie D, Cole S, Colston MJ, et al. Detection of rifampicin-resistance mutations in Mycobacterium tuberculosis. Lancet 1993;341:647-650. 17. Heep M, Brandstatter B, Rieger U, Lehn $\mathrm{N}$, Richter E, Rusch-Gerdes S, et al. Frequency of $r p \circ B$ mutations inside and outside the cluster I region in rifampin-resistant clinical Mycobacterium tuberculosis isolates. J Clin Microbiol 200 I;39:107-II0.

\section{Respuesta al comentario al artículo "Mutaciones asociadas con resistencia a rifampicina $\odot$ isoniazida en aislamientos clínicos de M. Tuberculosis de Sonora, México"}

Señor editor: Por este medio, los abajo firmantes y autores del trabajo titulado "Mutaciones asociadas con resistencia a rifampicina o isoniazida en aislamientos clínicos de M. tuberculosis de Sonora, Méxi$\mathrm{co}^{\prime \prime}$, le informamos que después de revisar y discutir la carta enviada por Flores-Tre- viño Samantha y Garza-González Elvira, así como la literatura correspondiente, expresamos nuestra aceptación a sus observaciones, por lo que en lo sucesivo utilizaremos ambas clasificaciones moleculares para la discusión de nuestros resultados. No obstante lo anterior, destacamos que la finalidad de nuestro trabajo, más allá de la intención de profundizar en la identificación de mutaciones específicas, fue iniciar la caracterización genotípica de cepas clínicas de M. tuberculosis resistentes a isoniazida o rifampicina aisladas en Sonora, México, ${ }^{1}$ principalmente para fortalecer el monitoreo de la tuberculosis multidrogorresistente ${ }^{2}$ en nuestro país. El otro aspecto relevante de nuestro trabajo es que al analizar seis aislamientos clínicos en los que no se obtuvo un perfil fenotípico de resistencia a fármacos, dos de ellos presentaron mutaciones asociadas con resistencia a isoniazida o rifampicina. De igual manera, destacamos que en cuatro de las cepas no se detectaron mutaciones asociadas con resistencia a esos fármacos, dentro de las regiones analizadas y más frecuentemente evaluadas en este tipo de estudios, ${ }^{3-5}$ incluso para el diseño de nuevos protocolos de evaluación de la farmacorresistencia en $M$. tuberculosis..$^{6-7}$ Finalmente, consideramos que la discusión y conclusiones de nuestro artículo son pertinentes ya que hemos identificado nuevas mutaciones en la región intergénica ahpC-oxyR, particularmente en una región (-12 a -17), región en donde se han detectado mutaciones asociadas con perfiles de resistencia a isoniazida. ${ }^{5}$ En virtud de lo antes expuesto, consideramos que es recomendable realizar la caracterización genotípica sistemática de todas las cepas clínicas de M. tuberculosis que presenten resistencia a cualquier fármaco antituberculoso, en Sonora, México y en otras regiones geográficas del país.

Enrique Bolado-Martínez, D en C(I) ebolado@guayacan.uson.mx Anxis Pérez-Mendoza, $M$ en $C_{\text {, }}^{(2)}$ Francisco Monserrat Alegria-Morquecho, QBC, (3) María del Carmen Candia-Plata, D en $C_{\text {, }}^{(4)}$ María del Rosario Aguayo-Verdugo, QB, (3) Gerardo Álvarez-Hernández, D en C. ${ }^{(4)}$

(I) Departamento de Ciencias Químico Biológicas,
Universidad de Sonora. Hermosillo, Sonora, México

(2) Doctorado Institucional en Ciencias de la Salud, Universidad Autónoma de Yucatán. Yucatán, México

(3) Laboratorio Estatal de Salud Pública. Hermosillo, Sonora. México.

(4) Departamento de Medicina y Ciencias de la Salud, Universidad de Sonora. Hermosillo, Sonora, México

\section{Referencias}

I. Bolado-Martinez E, Perez-Mendoza A, Alegria-Morquecho FM, Candia-Plata M del C, Aguayo-Verdugo M del R, Alvarez-Hernandez G. DNA mutations associated to rifampicin or isoniazid resistance in $\mathrm{M}$. tuberculosis clinical isolates from Sonora, Mexico. Salud Publica Mex 20I2;54:167-I70.

2. WHO. Implementing the Stop TB strategy: a handbook for national tuberculosis control programmes. Geneva: World Health Organization, 2008 (WHO/HTM/TB/2008.40I).

3. Luo T, Zhao M, Li X, Xu P, Gui X, Pickerill S, et $a$ l. Selection of mutations to detect multidrugresistant Mycobacterium tuberculosis strains in Shanghai, China. Antimicrob Agents Chemother 2010;54:1075-1081.

4. Kozhamkulov U, Akhmetova A, Rakhimova S, Belova E, Alenova A, Bismilda V, et al. Molecular characterization of rifampicin- and isoniazid-resistant Mycobacterium tuberculosis strains isolated in Kazakhstan. Jpn J Infect Dis 20I I;64:253-255.

5. Therese KL, Gayathri R, Balasubramanian S, Natrajan S, Madhavan HN. Phenotypic and genotypic characteristics of drug resistance in Mycobacterium tuberculosis isolates from pediatric population of Chennai, India. Indian J Med Microbiol 2012;30:4 II-4I7

6. Bang $\mathrm{H}$, Park S, Hwang J, Jin $\mathrm{H}$, Cho E, Kim DY, et al. Improved rapid molecular diagnosis of multidrug-resistant tuberculosis using a new reverse hybridization assay, REBA MTB-MDR. J Med Microbiol 201 I;60: I447- I454.

7. Daum LT, Rodriguez JD, Worthy SA, Ismail NA, Omar SV, Dreyer AW, et al. Next-generation ion torrent sequencing of drug resistance mutations in Mycobacterium tuberculosis Strains. J Clin Microbiol 2012;50:383I-3837.

\section{Letalidad por fiebre manchada por Rickettsia rickettsii en pacientes de un hospital pediátrico del estado de Sonora, 2004-20 I 2}

La fiebre manchada por Rickettsia rickettsii (FMRR) es la más letal de las infecciones del grupo de fiebres manchadas. ${ }^{1-2} \mathrm{Si}$ bien la enfermedad puede ocurrir en cualquier 